\title{
Construction of a cDNA library and preliminary analysis of the expressed sequence tags of the earthworm Eisenia fetida (Savigny, 1826)
}

\author{
CHANG LIU $^{1 *}$, XUAN WANG $^{1 *}$, JIU-BO TIAN $^{1}$, PEI-YUAN MA ${ }^{1}$, FAN-XIU MENG $^{1}$, \\ QI ZHANG ${ }^{1}$, BAO-FENG YU ${ }^{1}$, RUI GUO ${ }^{1}$, ZHI-ZHEN LIU ${ }^{1}$, HAI-LONG WANG ${ }^{1}$, JUN XIE ${ }^{1}$, \\ NIU-LIANG CHENG ${ }^{1}$, JIAN-HUA WANG ${ }^{2}$, BO NIU $^{1,2}$ and GONG-QIN SUN ${ }^{1,3}$
}

\author{
${ }^{1}$ Department of Biochemistry and Molecular Biology, Shanxi Medical University, Taiyuan, Shanxi 030001; \\ ${ }^{2}$ Department of Biotechnology, Beijing Municipal Key Laboratory of Child Development and Nutriomics, \\ Capital Institute of Pediatrics, Beijing 100020, P.R. China; ${ }^{3}$ Department of Cell and Molecular Biology, \\ University of Rhode Island, Kingston, RI 02881, USA
}

Received June 24, 2018; Accepted January 11, 2019

DOI: $10.3892 / \mathrm{mmr} .2019 .9938$

\begin{abstract}
Earthworms are useful indicator organisms of soil health and Eisenia fetida have been extensively used as test organisms in ecotoxicological studies. In order to gain insight into the gene expression profiles associated with physiological functions of earthworms, a full-length enriched cDNA library of the Eisenia fetida genome was successfully constructed using Switching Mechanism at 5'End of RNA Template technology. Construction of a cDNA library and analysis of Expressed Sequence Tags (ESTs) are efficient approaches for collecting genomic information and identifying genes important for a given biological process. Furthermore, analysis of the expression abundance of ESTs was performed with the aim of providing genetic and transcriptomic information on the development and regenerative process of earthworms. Phrep and Crossmatch were used to process EST data and a total of 1,140 high-quality EST sequences were determined by sequencing random cDNA clones from the library. Clustering
\end{abstract}

Correspondence to: Dr Bao-Feng Yu, Department of Biochemistry and Molecular Biology, Shanxi Medical University, 85 Jiefang South Road, Taiyuan, Shanxi 030001, P.R. China

E-mail: shanxiyangcheng@126.com

Dr Bo Niu, Department of Biotechnology, Beijing Municipal Key Laboratory of Child Development and Nutriomics, Capital Institute of Pediatrics, 56 Nanlishi Road, Xicheng, Beijing 100020, P.R. China E-mail: niub2004@126.com

${ }^{*}$ Contributed equally

Abbreviations: EST, expressed sequence tags; GO, Gene Ontology; KEGG, Kyoto Encyclopedia of Genes and Genomes

Key words: Earthworm Eisenia fetida (Savigny, 1826), cDNA library, expressed sequence tags, GO annotation, KEGG pathway analysis of sequences revealed a total of 593 unique sequences including 225 contiguous and 368 singleton sequences. Basic Local Alignment Search Tool analysis against the Kyoto Encyclopedia of Genes and Genomes database resulted in 593 significant hits ( $\left.\mathrm{P}-\mathrm{value}<1 \times 10^{-8}\right)$, of which 168 were annotated through Gene Ontology analysis. The STRING database was used to determine relationships among the 168 ESTs, identifying associated genes involved in protein-protein interactions and gene expression regulation. Based on nucleic acid and protein sequence homology, the mutual relationships between 287 genes could be obtained, which identified a portion of the ESTs as known genes. The present study reports on the construction of a high-quality cDNA library representative of adult earthworms, on a preliminary analysis of ESTs and on a putative functional analysis of ESTs. The present study is expected to enhance our understanding of the molecular basis underlying the biological development of earthworms.

\section{Introduction}

Earthworms are terrestrial annelids in the oligochaeta subclass, with a generally preferred habitat of damp and loose soil. They include $\sim 3,000$ species worldwide, with 229 species in China $(1,2)$. In a wide variety of soil types, earthworms serve vital roles in converting large pieces of organic matter into rich humus to enrich soil fertility. The earthworms are the highest evolutionary species capable of regenerating an anterior portion containing the central nerve system, heart and clitellum (3). The anterior regeneration is a unique developmental process that requires cell proliferation, re-differentiation and sophisticated cell-cell communication. This process can serve as a useful model for investigating normal development and differentiation (4).

Over the past several years, cDNA library construction and analysis have become established as indispensable methods for functional genome analysis since they provide detailed information about the genomic mechanisms underlying the diverse processes of an organism (5). However, conventionally 
generated cDNA libraries contain a high percentage of 5 '-truncated clones, limiting the utility of such libraries. The Switching Mechanism at 5'End of RNA Template (SMART) technique (6) amplifies and enriches the full-length mRNA, and thus generates cDNA libraries with a significantly improved ratio of full-length to partial cDNA sequences. In the present study, the SMART technique was adopted to construct a high quality library of full-length cDNAs representative of adult earthworms, namely of the earthworm Eisenia fetida (Savigny, 1826).

Unlike other model organisms, none of the oligochaete genomes have been sequenced to the best of our knowledge, and genomic research on earthworms lags behind that of other model species such as Mus musculus. In the absence of the full genome sequences, expressed sequence tags (ESTs) aid the rapid detection of expressed genes via sequence analysis, and are a significant resource for comparative and functional genomic studies (2). In addition, among the biological techniques for transcriptome analysis, the determination of ESTs is considered the simplest method for profiling the transcriptome, which is also particularly useful in the development of cDNA microarrays for systematic identification of differentially expressed genes (7). Analysis of ESTs is an effective method for rapidly analyzing gene expression, characterizing gene functions and discovering new genes that are important for specific developmental and physiological processes (8). The present study established 593 ESTs, representing 168 genes and 425 unknown tags, providing a gene expression profile of earthworm development. This collection of ESTs may provide a valuable basis for future research on the physiology of earthworms.

\section{Materials and methods}

Isolation of total RNA and mRNA. Eisenia fetida earthworms were purchased from Beijing Shuangqiao Farm (Beijing, China). Fully developed adult Eisenia fetida earthworms weighing 0.3-0.6 g (live weight) were selected for all experiments. All earthworm tissues were harvested and total RNA was isolated using TRIzol reagent (Invitrogen; Thermo Fisher Scientific, Inc., Waltham, MA, USA). The integrity of total RNA was analyzed by electrophoresis using $1 \%$ agarose gels. Isolation of poly(A) mRNA from total RNA was carried out using an MN-NucleoTrap ${ }^{\circledR}$ mRNA kit (Machery-Nagel GmbH \& Co. KG, Düren, Germany) according to the manufacturer's protocol. Oligo(dT) beads suspension was applied to total RNA and incubated at $68^{\circ} \mathrm{C}$ for 5 min before eluting mRNA. Then ethidium bromide (EB) staining was applied and $1 \%$ agarose gel was used to visualize the result. The isolated mRNA was further vacuum concentrated using Concentrator plus $^{\mathrm{TM}}$ (Eppendorf, Hamburg, Germany). The quantity and integrity of isolated mRNA were determined using a nanodrop spectrophotometer and agarose gel electrophoresis, respectively.

cDNA library construction. A total of $~ 8,048.4 \mathrm{ng}$ mRNA was used for single-stranded cDNA synthesis. The purified mRNA was used as a template, Oligod(T)18 with XhoI cleavage site was used as the primer, and first strand cDNA was transcribed at $42^{\circ} \mathrm{C}$ using SuperScript ${ }^{\mathrm{TM}}$ II RnaseH-Reverse
(Thermo Fisher Scientific, Inc.). Then the mRNA was digested using $\mathrm{RNaseH}$, and the resultant mRNA fragments were used as further primers. The first cDNA chain was used as a template for double-stranded cDNA synthesis, using DNA Polymerase I (Takara Biotechnology Co., Ltd., Beijing, China). The ends of the double-stranded cDNA were ligated by T4 DNA polymerase and the ligation products were purified by phenol/chloroform/isoamyl alcohol to remove excess impurities such as protein. Subsequently, the double-stranded DNA fragments were ligated into EcoRI Adaptor using T4 DNA ligase at $4^{\circ} \mathrm{C}$ overnight. Then the double-stranded DNA fragments were phosphorylated with T4 Polynucleotide Kinase and digested with XhoI. Following XhoI digestion of the double-stranded cDNA, producing XhoI sticky ends, a QIAEXII Gel Extraction kit (Beijing BioDev-Tech, Beijing, China) was used to recycle $0.5-4 \mathrm{~Kb}$ fragments. The recycled cDNA was preserved at $-20^{\circ} \mathrm{C}$. Then the cDNA was ligated into the pBluescript II SK(+) XR vector (Promega Corporation, Madison, WI, USA) in a 3:1 molar ratio with T4 DNA ligase at $4^{\circ} \mathrm{C}$ overnight. To reduce the redundancy and avoid the underrepresentation of different transcript species, cDNA fragments with different fractionated sizes were balanced and subjected to library construction $(9,10)$. Prior to transformation, mixing of all ligated products with microporous membranes was performed to remove salt ions. Subsequently the products were transformed into $5 \times 10^{7} / \mathrm{ml}$ DH10B competent cells (Thermo Fisher Scientific, Inc.), plated on agar plates (10 cm diameter) by pipetting the cells onto the middle of the plate and spreading, and monoclonal colonies were selected for PCR amplification. The inserted sequences in the plasmids were amplified by PCR using T3 primers (5'-ATTAACCCTCACTAAAGGGA-3') and T7 primers (5'-TAATACGACTCACTATAGGG-3'). The total volume of PCR reaction mixture was $20 \mu \mathrm{l}$, containing $1 \mu \mathrm{l}$ template, $10 \mu 1$ 2XTaq MasterMix (CWBIO, Beijing, China), $1 \mu \mathrm{l}$ T3 primers (10 pmol), $1 \mu \mathrm{l} \mathrm{T} 7$ primers (10 pmol) and $7 \mu 1$ $\mathrm{ddH}_{2} \mathrm{O}(\mathrm{CWBIO})$. Cycling conditions were: $94^{\circ} \mathrm{C}$ for $5 \mathrm{~min}$, followed by 30 cycles of $94^{\circ} \mathrm{C}$ for $30 \mathrm{sec}, 55^{\circ} \mathrm{C}$ for $40 \mathrm{sec}$ and $72^{\circ} \mathrm{C}$ for $60 \mathrm{sec}$, followed by $72^{\circ} \mathrm{C}$ for $5 \mathrm{~min}$.

Bioinformatic analysis. cDNA clones were selected randomly from the cDNA library and the vector sequences were trimmed from the raw sequence data using Vecscreen tool (www.ncbi.nlm.nih.gov/tools/vecscreen/) from the National Center for Biotechnology Information. The sequence of each EST was also edited, mainly to remove ambiguous bases and poor-quality sequences (nucleotide sequences $<100$ bp). All edited sequences were assembled into groups using SeqMan software version 8.0 (DNASTAR, Madison, WI, USA). The processed cDNA sequences were used to perform a BLAST search in the GenBank database to compare all available ESTs and genes to date (11). The Basic Local Alignment Search Tool (BLAST; blast.ncbi.nlm.nih.gov/Blast.cgi) results with $\mathrm{P}$-values $<1 \times 10^{-8}$ were generally regarded as a significant match $(12,13)$. A large-scale Unigene assembly of the ESTs was initiated to identify and functionally annotate as many unique transcripts as possible. BLAST analysis against the Kyoto Encyclopedia of Genes and Genomes (KEGG) database, and protein and nucleic acid databases was conducted for examination of biological functions. The ESTs homologous to known proteins were further annotated for Gene Ontology 
(GO; www.geneontology.org) terms and the GO analysis was carried out using WEB-based GEne SeT AnaLysis Toolkit (WebGestalt; www.webgestalt.org/option.php) (14,15).

\section{Results}

Construction of cDNA library. Obtaining an adequate quantity of high quality mRNA initially is the key to yielding a sufficient quantity of first-strand full-length cDNA by reverse transcription. In the present study, total RNA was extracted from the tissues of earthworms. As shown in Fig. 1, 28s and 18s bands were clearly visible in the electrophoresis gel of total RNA, indicating that the total RNA was obtained. The optical density (OD) ${ }_{260} / \mathrm{OD}_{280}$ ratio for the total RNA was 2.04, well within the range of 1.8-2.1, indicating that the isolated total RNA was suitable for cDNA library construction.

Once the double-stranded cDNA was synthesized as described in the Materials and methods, the present study determined the size distribution of the products. Diffuse strips between $0.5-4.0 \mathrm{~kb}$ could be detected by $1 \%$ agarose gel electrophoresis, which indicated that double-stranded cDNAs were successfully synthesized. A cDNA library of $4.12 \times 10^{5}$ clones was obtained and half of the bacteria were cultured for amplification, which produced a total of $1.4 \times 10^{11}$ clones. Several colonies were selected, and the inserted sequences in the plasmids were amplified by PCR using T3 and T7 primers. The PCR products were detected using $1 \%$ agarose gel electrophoresis as clear bands. No nonspecific bands were identified and the recombinant rate was $97 \%$ (Fig. 2).

To investigate the quality of the full-length cDNA library, the lengths of the cDNA inserts were assessed. Sequence outputs were manually edited to remove vector and ambiguous sequences. Then, the sequence data of the cDNA clones obtained by random partial sequencing were searched in the NCBI GenBank using BLAST to identify similarities with sequences in the nucleic acid databases. An evaluation of cDNA insert size and its distribution revealed a low level of insert size bias in the final cDNA library. The majority of the cDNA inserts were larger than $500 \mathrm{bp}$.

EST analysis. Instead of the amplified library, the primary cDNA library was used to generate ESTs to reduce the redundancy of cDNA clones. Following the removal of the redundant sequences and low-quality sequences (<100 bp), 1,148 effective sequences $(>100 \mathrm{bp}$ ) from the total cDNA sequences were obtained. As shown in Fig. 3, 53 ESTs were 100-300 bp, 261 ESTs were 300-500 bp, 828 ESTs were 500-700 bp and 6 ESTs were larger than $700 \mathrm{bp}$. Taken together, 1,148 ESTs were larger than $100 \mathrm{bp}$. Among them, the shortest sequence was $100 \mathrm{bp}$, the longest was $718 \mathrm{bp}$ and the average length was $\sim 452$ bp. Following sequencing, a homology BLAST search and assembling of the data, 368 singletons and 225 contigs were obtained out of the 1,140 high-quality ESTs, as shown in Table I. Additionally, a total of 593 individual ESTs were analyzed and 168 ESTs annotated in GenBank with nematode homology (Table I).

GO annotation and bioinformatic analysis. The cDNA functions were classified using the GO database into the three
Table I. Summary of ESTs obtained from the cDNA library of earthworms.

\begin{tabular}{lr}
\hline ESTs & Number \\
\hline Total number of ESTs & 1,256 \\
Total length of ESTs (bp) & 568,140 \\
Average length of ESTs (bp) & 452.34 \\
Unique genes & 593 \\
Contigs & 225 \\
Singletons & 368 \\
Annotation & 168
\end{tabular}

ESTs, expressed sequence tags.

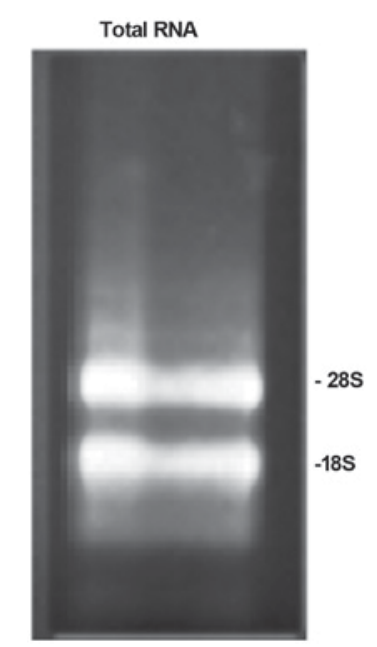

Figure 1. Result of the electrophoresis of total RNA. A total of 1,056 $\mu \mathrm{g}$ RNA was obtained from the tissues of earthworms. The optical density values were $\mathrm{A}_{260}=0.880$ and $\mathrm{A}_{280}=0.430 ; \mathrm{A}_{260} / \mathrm{A}_{280}=2.04$.

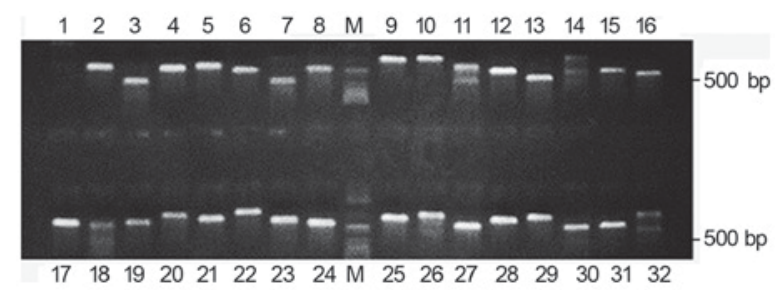

Figure 2. Amplified inserts of cDNA clones from the constructed cDNA library. M, DNA marker; Lanes 1-32, insert cDNA (>500 bp) clones from phage plaques.

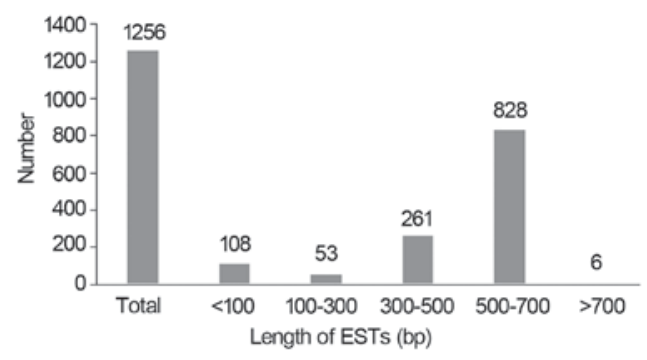

Figure 3. Different groups of ESTs, separated by length. ESTs, expressed sequence tags. 
A

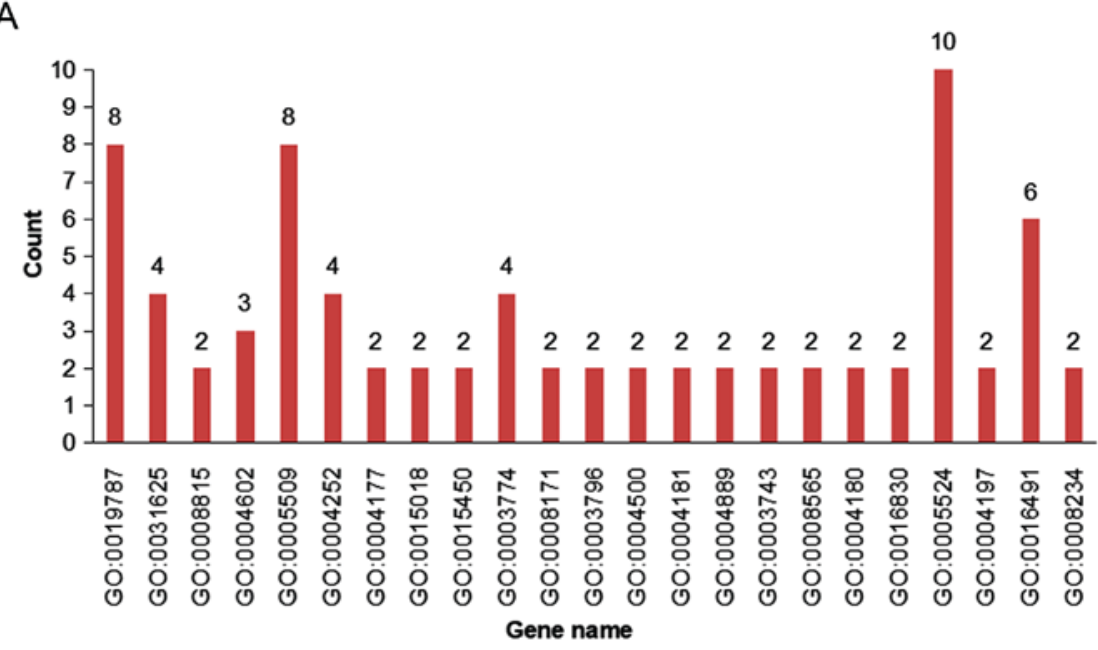

B
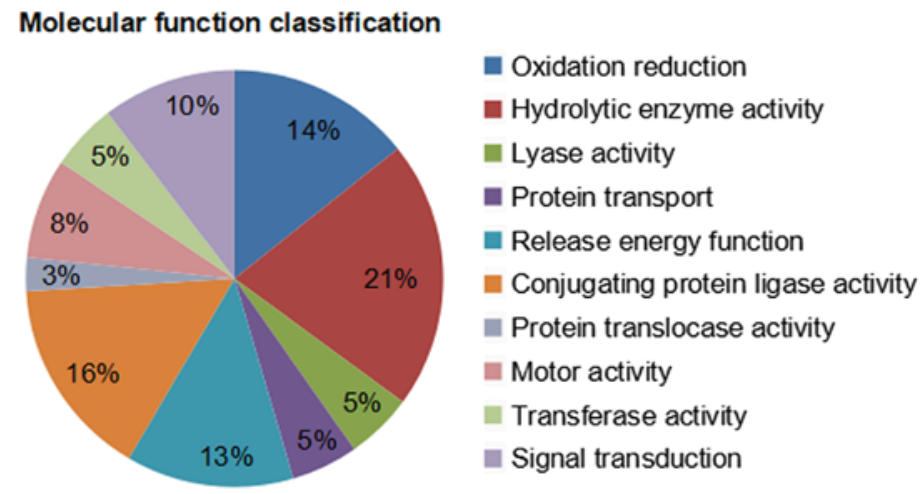

Figure 4. Distribution of GO molecular functions. (A) The distribution of 77 expressed sequence tags with molecular functions. (B) The classification of identified genes based on the relevant molecular functions. GO, Gene Oncology.

main categories of molecular functions, cell components and biological processes.

GO annotation of genes associated with molecular functions indicated that among the 168 ESTs, 46\% (77/168 ESTs) were associated with growth and metabolic pathways, with the distribution of the 77 ESTs shown in Fig. 4A. Out of the 77 ESTs, 21\% (16 ESTs) were associated with 'proteolytic enzymes', 16\% (12 ESTs) with 'protein ligases', 14\% (11 ESTs) with 'oxido-reductases', $13 \%$ (10 ESTs) with 'energy release', $10 \%$ (8 ESTs) with 'signal transduction and cell communication', 5\% (4 ESTs) with 'transport' and only 3\% (2 ESTs) with 'post-translational modification', 'protein turnover' and 'chaperones' (Fig. 4B).

Cellular components associated with the cDNAs included 'myosin', the 'citrate lyase compound', the 'mitochondrial inner membrane translocase compound', 'microtubules', the 'mitochondrial inner membrane' and 'nucleosomes'. The proportions of cellular components are presented in Fig. 5. It can be observed that the proportion of 'myosin' among cellular components was the largest. Myosin, an actin-dependent molecular motor, is involved in a number of important functions in earthworms. In particular, the myosin network can drive movement and support different moving speeds of earthworms. This specific feature is closely related to the free moving ability of earthworms (16).
Regarding biological processes, known genes were determined as those presenting significant matches to protein sequences with known functions in non-redundant nucleotide databases. According to these biological functions, the biological processes component was divided into different functions including 'larval development' (46\%), 'changes of cell morphogenesis' (6\%), 'the process of cytokinesis' $(12 \%)$, 'post-translational protein modification' $(15 \%)$, 'stress response' (5\%), 'cell redox homeostasis' (2\%), 'protein polymerization' (2\%) and 'protein catabolism' (12\%), as shown in Fig. 6. It was concluded from the above data that promoting growth was considered to be an important biological function of genes associated with biological processes.

KEGG pathway annotation. KEGG is a collection of online databases describing pathways associated with biochemical, genomic and enzymatic processes. Furthermore, it provides annotations of biochemical pathways for the species in which the genome has been sequenced (17). In this analysis, proteins are not viewed as individual gene products but are organized into pathways and networks according to their biological function(s). In the present study, from the data in Table II, 15 of the 168 ESTs were revealed to be involved in metabolism. Notably, 4 of the 15 ESTs (27\%) were involved in the ubiquitin-mediated proteolysis pathway, which was the most 
Table II. Pathway analysis based on the Kyoto Encyclopedia of Genes and Genomes database.

\begin{tabular}{|c|c|c|c|c|c|}
\hline Pathway & Count & P-value & Q-value & Protein & Input symbol \\
\hline Ubiquitin-mediated proteolysis & 4 & $1.12 \times 10^{-5}$ & $1.73 \times 10^{-4}$ & $\begin{array}{l}\text { ubc-14;ubc-13; } \\
\text { ubc-18;let-70 }\end{array}$ & $\begin{array}{l}\text { Y87G2A.9;Y54G2A.31; } \\
\text { R01H2.6;M7.1 }\end{array}$ \\
\hline Glutathione metabolism & 3 & $2.55 \times 10^{-5}$ & $2.63 \times 10^{-4}$ & $\begin{array}{l}\text { W07G4.4;F26E4.12; } \\
\text { C11E4.1 }\end{array}$ & $\begin{array}{l}\text { W07G4.4;F26E4.12; } \\
\text { C11E4.1 }\end{array}$ \\
\hline Arachidonic acid metabolism & 2 & $2.01 \times 10^{-4}$ & $1.25 \times 10^{-3}$ & F26E4.12;C11E4.1 & F26E4.12;C11E4.1 \\
\hline Chondroitin sulfate biosynthesis & 1 & $6.54 \times 10^{-3}$ & $2.03 \times 10^{-2}$ & sqv-8 & ZK1307.5 \\
\hline Heparan sulfate biosynthesis & 1 & $1.14 \times 10^{-2}$ & $2.75 \times 10^{-2}$ & sqv-8 & ZK1307.5 \\
\hline Riboflavin metabolism & 1 & $1.14 \times 10^{-2}$ & $2.75 \times 10^{-2}$ & F02E9.7 & F02E9.7 \\
\hline Selenoamino acid metabolism & 1 & $3.39 \times 10^{-2}$ & $4.62 \times 10^{-2}$ & seld-1 & Y45F10A.4 \\
\hline$\gamma$-hexachlorocyclohexane degradation & 1 & $4.02 \times 10^{-2}$ & $4.62 \times 10^{-2}$ & F02E9.7 & F02E9.7 \\
\hline Fructose and mannose metabolism & 1 & $4.18 \times 10^{-2}$ & $4.62 \times 10^{-2}$ & R04B5.5 & R04B5.5 \\
\hline
\end{tabular}

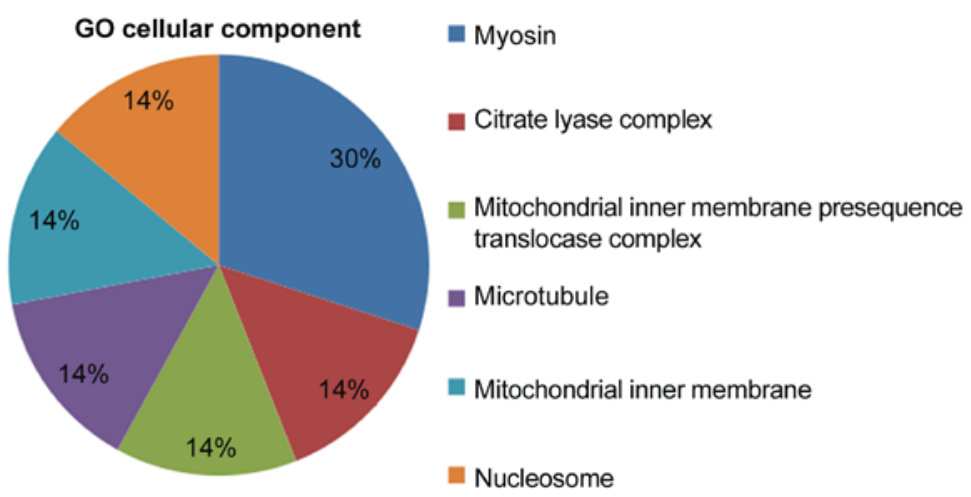

Figure 5. Distribution of GO cellular components. GO, Gene Oncology.

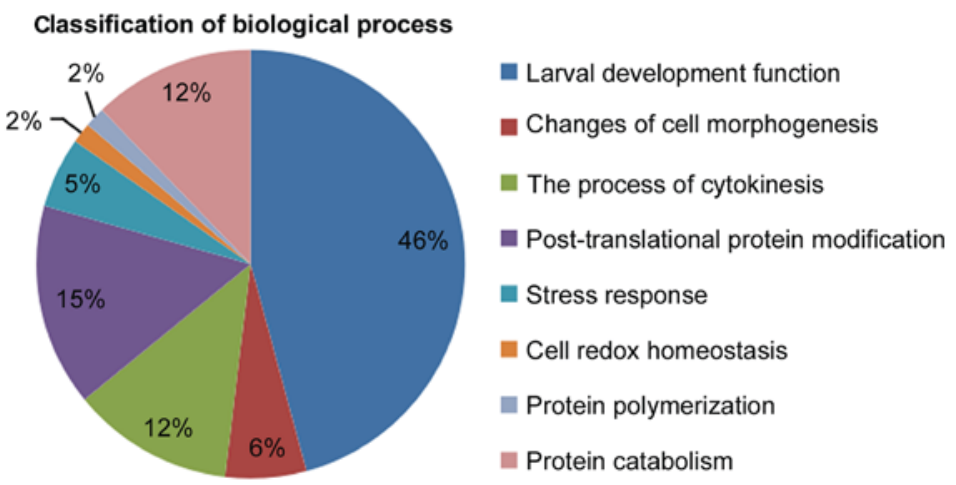

Figure 6. Classification of identified genes based on relevant biological processes.

represented. The glutathione metabolism (3 ESTs) and arachidonic acid metabolism (2 ESTs) pathways were the second and third most represented pathways, respectively. Additionally, chondroitin sulfate biosynthesis, heparan sulfate biosynthesis, riboflavin metabolism, selenoamino acid metabolism, $\gamma$-hexachlorocyclohexane degradation, and the fructose and mannose metabolism pathways were also represented.

Mutual relationship between 287 genes. Relationships among the 168 ESTs were analyzed using the STRING database, and a functional association network was determined with
287 nodes, as shown in Table III. Each node corresponds to a gene and each (weighted) edge represents the evidence of a functional association between the gene pair. Predicted potential regulators are presented in Fig. 7. It can be seen from the STRING results that the most associated nodes (blue) included the 19 genes: Ribosomal protein L (rpl)-1, ribosomal protein $\mathrm{S}$ (rps)-0, rpl-4, rpl-5, rps-13, rps-2, acidic ribosomal protein (rla)-1, translocon-associated protein-4, transcription factor BTF3 homolog, rpl-7A, iff-1, rps-17, elongation factor $1 \alpha 3$, polyadenylate-binding protein-1, $\mathrm{rps}-28$, translationally-controlled tumor protein homolog-1, rpl-18, 
Table III. Relationships between the 287 associated genes, including several important parameters.

\begin{tabular}{|c|c|c|c|c|c|c|c|}
\hline node1 & node2 & $\begin{array}{l}\text { node1_- } \\
\text { string_id }\end{array}$ & $\begin{array}{l}\text { node2_- } \\
\text { string_id }\end{array}$ & $\begin{array}{c}\text { node1__ } \\
\text { external_id }\end{array}$ & $\begin{array}{c}\text { node2_- } \\
\text { external_id }\end{array}$ & coexpression & $\begin{array}{l}\text { combined } \\
\text { score }\end{array}$ \\
\hline rpl-5 & atp-2 & 502,561 & 496,479 & F54C9.5.1 & C34E10.6.3 & 0.866 & 0.871 \\
\hline rpl-18 & snr-2 & 510,406 & 508,903 & Y45F10D.12.2 & W08E3.1 & 0.426 & 0.468 \\
\hline trap-4 & rps-2 & 511,330 & 497,379 & Y56A3A.21.2 & С49H3.11.1 & 0.243 & 0.919 \\
\hline sod-2 & daf-21 & 498,735 & 497,248 & F10D11.1.1 & C47E8.5.1 & 0 & 0.572 \\
\hline rla-2 & rps-4 & 511,588 & 510,280 & Y62E10A.1.1 & Y43B11AR.4.2 & 0.981 & 0.998 \\
\hline rpl-1 & drs-1 & 511,889 & 493,869 & Y71F9AL.13a.1 & B0464.1.1 & 0.492 & 0.523 \\
\hline skr-1 & sdhd-1 & 501,798 & 500,588 & F46A9.5.3 & F33A8.5.2 & 0 & 0.587 \\
\hline $\operatorname{prdx}-6$ & sod-2 & 509,858 & 498,735 & Y38C1AA.11 & F10D11.1.1 & 0.233 & 0.726 \\
\hline act-4 & rps-0 & 505,013 & 493,798 & $\mathrm{M} 03 \mathrm{~F} 4.2 \mathrm{a}$ & B0393.1.1 & 0.426 & 0.425 \\
\hline daf-21 & rps-13 & 497,248 & 495,308 & C47E8.5.1 & C16A3.9.1 & 0.402 & 0.474 \\
\hline rpl-1 & rpl-4 & 511,889 & 493,528 & Y71F9AL.13a.1 & B0041.4.1 & 0.8 & 0.999 \\
\hline cyc-1 & atp-2 & 497,719 & 496,479 & C54G4.8.1 & C34E10.6.3 & 0.849 & 0.873 \\
\hline rla-1 & act-4 & 509,817 & 505,013 & Y37E3.7.2 & M03F4.2a & 0.538 & 0.538 \\
\hline rpl-7A & rps-17 & 509,604 & 506,860 & Y24D9A.4a & T08B2.10.1 & 0.872 & 0.923 \\
\hline rps-28 & rpl-5 & 510,228 & 502,561 & Y41D4B.5.2 & F54C9.5.1 & 0.77 & 0.887 \\
\hline rpl-18 & cyc-1 & 510,406 & 497,719 & Y45F10D.12.2 & C54G4.8.1 & 0.387 & 0.411 \\
\hline tpi-1 & rps-0 & 509,457 & 493,798 & Y17G7B.7.2 & B0393.1.1 & 0.477 & 0.49 \\
\hline daf-21 & drs-1 & 497,248 & 493,869 & C47E8.5.1 & В0464.1.1 & 0.281 & 0.543 \\
\hline $1 \mathrm{sm}-3$ & rla-2 & 511,591 & 511,588 & Y62E10A.12.2 & Y62E10A.1.1 & 0 & 0.408 \\
\hline rpl-1 & rps-4 & 511,889 & 510,280 & Y71F9AL.13a.1 & Y43B11AR.4.2 & 0.819 & 0.989 \\
\hline tpi-1 & atp-2 & 509,457 & 496,479 & Y17G7B.7.2 & C34E10.6.3 & 0.448 & 0.877 \\
\hline lev-11 & unc-60 & 509,147 & 496,717 & Y105E8B.1d & C38C3.5b.1 & 0 & 0.431 \\
\hline rpl-5 & icd-1 & 502,561 & 497,806 & F54C9.5.1 & С56C10.8.1 & 0.864 & 0.87 \\
\hline rla-2 & pab-1 & 511,588 & 509,182 & Y62E10A.1.1 & Y106G6H.2a.4 & 0.102 & 0.904 \\
\hline unc-54 & mlc-3 & 498,853 & 498,666 & F11C3.3.1 & F09F7.2a.2 & 0.452 & 0.452 \\
\hline alp-1 & cyc-1 & 507,123 & 497,719 & $\mathrm{~T} 11 \mathrm{~B} 7.4 \mathrm{~d}$ & C54G4.8.1 & 0 & 0.409 \\
\hline mca-3 & cmd-1 & 511,739 & 507,723 & Y67D8C.10b & T21H3.3.1 & 0 & 0.581 \\
\hline cyc-1 & rps-2 & 497,719 & 497,379 & C54G4.8.1 & С49H3.11.1 & 0.579 & 0.579 \\
\hline rpl-1 & daf-21 & 511,889 & 497,248 & Y71F9AL.13a.1 & C47E8.5.1 & 0.251 & 0.415 \\
\hline rla-2 & iff-1 & 511,588 & 506,602 & Y62E10A.1.1 & T05G5.10 & 0.436 & 0.521 \\
\hline rpl-1 & rla-1 & 511,889 & 509,817 & Y71F9AL.13a.1 & Y37E3.7.2 & 0.794 & 0.997 \\
\hline tpi-1 & vha-4 & 509,457 & 506,234 & Y17G7B.7.2 & T01H3.1.1 & 0.483 & 0.483 \\
\hline vha-4 & atp-2 & 506,234 & 496,479 & T01H3.1.1 & C34E10.6.3 & 0.304 & 0.724 \\
\hline tpi-1 & sod-2 & 509,457 & 498,735 & Y17G7B.7.2 & F10D11.1.1 & 0.213 & 0.84 \\
\hline rps-4 & act-4 & 510,280 & 505,013 & Y43B11AR.4.2 & M03F4.2a & 0.429 & 0.469 \\
\hline rpl-18 & rps-2 & 510,406 & 497,379 & Y45F10D.12.2 & С49H3.11.1 & 0.999 & 0.999 \\
\hline rpl-18 & rps-13 & 510,406 & 495,308 & Y45F10D.12.2 & C16A3.9.1 & 0.999 & 0.999 \\
\hline rps-17 & tct-1 & 506,860 & 499,979 & T08B2.10.1 & F25H2.11.2 & 0.874 & 0.884 \\
\hline nmy-1 & mlc-4 & 502,225 & 497,828 & F52B10.1 & C56G7.1.2 & 0 & 0.698 \\
\hline drs-1 & rpl-4 & 493,869 & 493,528 & B0464.1.1 & B0041.4.1 & 0.581 & 0.74 \\
\hline rpl-5 & rps-0 & 502,561 & 493,798 & F54C9.5.1 & B0393.1.1 & 0.999 & 0.999 \\
\hline sdhd-1 & cey-1 & 500,588 & 500,586 & F33A8.5.2 & F33A8.3.2 & 0.224 & 0.624 \\
\hline rpl-18 & rps-28 & 510,406 & 510,228 & Y45F10D.12.2 & Y41D4B.5.2 & 0.78 & 0.928 \\
\hline rps-28 & rps-13 & 510,228 & 495,308 & Y41D4B.5.2 & C16A3.9.1 & 0.794 & 0.979 \\
\hline pab-1 & rpl-5 & 509,182 & 502,561 & Y106G6H.2a.4 & F54C9.5.1 & 0.762 & 0.978 \\
\hline rps-4 & icd-1 & 510,280 & 497,806 & Y43B11AR.4.2 & С56C10.8.1 & 0.826 & 0.833 \\
\hline eft-3 & atp-2 & 505,324 & 496,479 & R03G5.1a.2 & C34E10.6.3 & 0.478 & 0.602 \\
\hline iff-1 & rps-0 & 506,602 & 493,798 & T05G5.10 & B0393.1.1 & 0.604 & 0.623 \\
\hline rpl-18 & pab-1 & 510,406 & 509,182 & Y45F10D.12.2 & Y106G6H.2a.4 & 0.522 & 0.95 \\
\hline iff-1 & rpl-4 & 506,602 & 493,528 & T05G5.10 & B0041.4.1 & 0.603 & 0.656 \\
\hline rla-2 & rpl-18 & 511,588 & 510,406 & Y62E10A.1.1 & Y45F10D.12.2 & 0.967 & 0.997 \\
\hline
\end{tabular}


Table III. Continued.

\begin{tabular}{|c|c|c|c|c|c|c|c|}
\hline node1 & node 2 & $\begin{array}{l}\text { node1_} \\
\text { string_id }\end{array}$ & $\begin{array}{l}\text { node2_- } \\
\text { string_id }\end{array}$ & $\begin{array}{c}\text { node1_- } \\
\text { external_id }\end{array}$ & $\begin{array}{c}\text { node2_- } \\
\text { external_id }\end{array}$ & coexpression & $\begin{array}{l}\text { combined } \\
\text { score }\end{array}$ \\
\hline crt-1 & $\mathrm{rpl}-5$ & 509,852 & 502,561 & Y38A10A.5.1 & F54C9.5.1 & 0.452 & 0.465 \\
\hline rps-28 & tpi-1 & 510,228 & 509,457 & Y41D4B.5.2 & Y17G7B.7.2 & 0.278 & 0.451 \\
\hline rps-4 & iff-1 & 510,280 & 506,602 & Y43B11AR.4.2 & T05G5.10 & 0.64 & 0.888 \\
\hline icd-1 & rps-13 & 497,806 & 495,308 & C56C10.8.1 & C16A3.9.1 & 0.856 & 0.856 \\
\hline trap-4 & rla-1 & 511,330 & 509,817 & Y56A3A.21.2 & Y37E3.7.2 & 0.176 & 0.923 \\
\hline nmy-1 & unc-54 & 502,225 & 498,853 & F52B10.1 & F11C3.3.1 & 0.102 & 0.565 \\
\hline trap-4 & rps-13 & 511,330 & 495,308 & Y56A3A.21.2 & C16A3.9.1 & 0.148 & 0.942 \\
\hline iff-1 & rps-13 & 506,602 & 495,308 & T05G5.10 & C16A3.9.1 & 0.614 & 0.614 \\
\hline maoc-1 & $\mathrm{ftn}-2$ & 498,218 & 497,902 & E04F6.3 & D1037.3.4 & 0.997 & 0.996 \\
\hline rpl-18 & eft-3 & 510,406 & 505,324 & Y45F10D.12.2 & R03G5.1a.2 & 0.428 & 0.938 \\
\hline exos-2 & cpf-1 & 512,052 & 500,247 & Y73B6BL.3 & F28C6.3 & 0.403 & 0.403 \\
\hline rla-2 & rla-1 & 511,588 & 509,817 & Y62E10A.1.1 & Y37E3.7.2 & 0.999 & 0.999 \\
\hline rla-2 & eft-3 & 511,588 & 505,324 & Y62E10A.1.1 & R03G5.1a.2 & 0.104 & 0.91 \\
\hline iff-1 & rps-2 & 506,602 & 497,379 & T05G5.10 & С49H3.11.1 & 0.696 & 0.903 \\
\hline rpl-7A & $\mathrm{rpl}-4$ & 509,604 & 493,528 & Y24D9A.4a & B0041.4.1 & 0.955 & 0.996 \\
\hline tpi-1 & daf-21 & 509,457 & 497,248 & Y17G7B.7.2 & C47E8.5.1 & 0.313 & 0.404 \\
\hline crt-1 & rps-0 & 509,852 & 493,798 & Y38A10A.5.1 & B0393.1.1 & 0.402 & 0.462 \\
\hline rpl-7A & iff-1 & 509,604 & 506,602 & Y24D9A.4a & T05G5.10 & 0.477 & 0.536 \\
\hline pab-1 & rps-17 & 509,182 & 506,860 & Y106G6H.2a.4 & T08B2.10.1 & 0.217 & 0.936 \\
\hline tpi-1 & rps-17 & 509,457 & 506,860 & Y17G7B.7.2 & T08B2.10.1 & 0.341 & 0.412 \\
\hline act-4 & rpl-4 & 505,013 & 493,528 & M03F4.2a & B0041.4.1 & 0.471 & 0.572 \\
\hline crt-1 & rps-2 & 509,852 & 497,379 & Y38A10A.5.1 & С49H3.11.1 & 0.507 & 0.523 \\
\hline rpl-7A & tct-1 & 509,604 & 499,979 & Y24D9A.4a & F25H2.11.2 & 0.793 & 0.794 \\
\hline exos-2 & lsm-3 & 512,052 & 511,591 & Y73B6BL.3 & Y62E10A.12.2 & 0.164 & 0.449 \\
\hline rla-1 & icd-1 & 509,817 & 497,806 & Y37E3.7.2 & C56C10.8.1 & 0.835 & 0.835 \\
\hline crt-1 & cmd-1 & 509,852 & 507,723 & Y38A10A.5.1 & T21H3.3.1 & 0.116 & 0.609 \\
\hline pab-1 & atp-2 & 509,182 & 496,479 & Y106G6H.2a.4 & C34E10.6.3 & 0.432 & 0.432 \\
\hline inf-1 & rpl-5 & 503,072 & 502,561 & F57B9.6a.3 & F54C9.5.1 & 0.409 & 0.409 \\
\hline trap-4 & rps-0 & 511,330 & 493,798 & Y56A3A.21.2 & B0393.1.1 & 0.174 & 0.911 \\
\hline rpl-18 & atp-2 & 510,406 & 496,479 & Y45F10D.12.2 & C34E10.6.3 & 0.476 & 0.659 \\
\hline rps-2 & drs- 1 & 497,379 & 493,869 & С49H3.11.1 & B0464.1.1 & 0.664 & 0.663 \\
\hline rpl-5 & drs-1 & 502,561 & 493,869 & F54C9.5.1 & В0464.1.1 & 0.332 & 0.403 \\
\hline rpl-5 & daf-21 & 502,561 & 497,248 & F54C9.5.1 & C47E8.5.1 & 0.559 & 0.676 \\
\hline rpl-18 & rpl-5 & 510,406 & 502,561 & Y45F10D.12.2 & F54C9.5.1 & 0.999 & 0.999 \\
\hline trap-4 & $\mathrm{rpl}-5$ & 511,330 & 502,561 & Y56A3A.21.2 & F54C9.5.1 & 0.273 & 0.949 \\
\hline snr-2 & rps-0 & 508,903 & 493,798 & W08E3.1 & B0393.1.1 & 0.35 & 0.414 \\
\hline unc-54 & daf-21 & 498,853 & 497,248 & F11C3.3.1 & C47E8.5.1 & 0 & 0.674 \\
\hline rpl-18 & rps-0 & 510,406 & 493,798 & Y45F10D.12.2 & B0393.1.1 & 0.999 & 0.999 \\
\hline rpl-7A & icd-1 & 509,604 & 497,806 & Y24D9A.4a & С56C10.8.1 & 0.737 & 0.756 \\
\hline tpi-1 & rps-2 & 509,457 & 497,379 & Y17G7B.7.2 & С49H3.11.1 & 0.484 & 0.521 \\
\hline qua-1 & nas-4 & 506,538 & 494,442 & T05C12.10 & C05D11.6 & 0.42 & 0.419 \\
\hline try-1 & cpn-1 & 513,205 & 501,580 & ZK546.15 & F43G9.9.1 & 0.408 & 0.408 \\
\hline rla-1 & iff-1 & 509,817 & 506,602 & Y37E3.7.2 & T05G5.10 & 0.551 & 0.691 \\
\hline rpl-18 & iff-1 & 510,406 & 506,602 & Y45F10D.12.2 & T05G5.10 & 0.564 & 0.577 \\
\hline pab-1 & eft-3 & 509,182 & 505,324 & Y106G6H.2a.4 & R03G5.1a.2 & 0.398 & 0.708 \\
\hline abcf-2 & $\mathrm{rps}-2$ & 508,270 & 497,379 & T27E9.7.1 & С49H3.11.1 & 0.268 & 0.452 \\
\hline tct-1 & rps-0 & 499,979 & 493,798 & F25H2.11.2 & B0393.1.1 & 0.987 & 0.987 \\
\hline alp-1 & daf-21 & 507,123 & 497,248 & T11B7.4d & C47E8.5.1 & 0 & 0.998 \\
\hline daf-21 & rps-0 & 497,248 & 493,798 & C47E8.5.1 & B0393.1.1 & 0.44 & 0.445 \\
\hline lev-11 & act-4 & 509,147 & 505,013 & Y105E8B.1d & M03F4.2a & 0.244 & 0.441 \\
\hline daf-21 & atp-2 & 497,248 & 496,479 & C47E8.5.1 & C34E10.6.3 & 0.415 & 0.745 \\
\hline
\end{tabular}


Table III. Continued.

\begin{tabular}{|c|c|c|c|c|c|c|c|}
\hline node 1 & node 2 & $\begin{array}{l}\text { node1_} \\
\text { string_id }\end{array}$ & $\begin{array}{l}\text { node2_- } \\
\text { string_id }\end{array}$ & $\begin{array}{c}\text { node1__ } \\
\text { external_id }\end{array}$ & $\begin{array}{c}\text { node2_- } \\
\text { external_id }\end{array}$ & coexpression & $\begin{array}{l}\text { combined } \\
\text { score }\end{array}$ \\
\hline rla-1 & tct-1 & 509,817 & 499,979 & Y37E3.7.2 & F25H2.11.2 & 0.998 & 0.999 \\
\hline rps-17 & iff-1 & 506,860 & 506,602 & T08B2.10.1 & T05G5.10 & 0.569 & 0.678 \\
\hline rps-28 & rla-1 & 510,228 & 509,817 & Y41D4B.5.2 & Y37E3.7.2 & 0.785 & 0.963 \\
\hline try-1 & сур-31A2 & 513,205 & 503,484 & ZK546.15 & H02I12.8 & 0.543 & 0.543 \\
\hline rpl-1 & rpl-5 & 511,889 & 502,561 & Y71F9AL.13a.1 & F54C9.5.1 & 0.799 & 0.995 \\
\hline rps-28 & rps-0 & 510,228 & 493,798 & Y41D4B.5.2 & B0393.1.1 & 0.76 & 0.966 \\
\hline act-4 & unc-54 & 505,013 & 498,853 & $\mathrm{M} 03 \mathrm{~F} 4.2 \mathrm{a}$ & F11C3.3.1 & 0.168 & 0.474 \\
\hline rpl-5 & tct-1 & 502,561 & 499,979 & F54C9.5.1 & F25H2.11.2 & 0.996 & 0.996 \\
\hline rla-1 & rps-13 & 509817 & 495,308 & Y37E3.7.2 & C16A3.9.1 & 0.999 & 0.999 \\
\hline rpl-1 & rps-17 & 511,889 & 5068,60 & Y71F9AL.13a.1 & T08B2.10.1 & 0.799 & 0.993 \\
\hline rpl-1 & rla-2 & 511,889 & 511,588 & Y71F9AL.13a.1 & Y62E10A.1.1 & 0.772 & 0.996 \\
\hline cey-1 & $\mathrm{cpf}-1$ & 500,586 & 500,247 & F33A8.3.2 & F28C6.3 & 0 & 0.904 \\
\hline pab-1 & rps-13 & 509,182 & 495,308 & Y106G6H.2a.4 & C16A3.9.1 & 0.327 & 0.928 \\
\hline sqv-8 & ret-1 & 512,932 & 508,785 & ZK1307.5 & W06A7.3f & 0 & 0.579 \\
\hline rps-28 & rpl-7A & 510,228 & 509,604 & Y41D4B.5.2 & Y24D9A.4a & 0.752 & 0.838 \\
\hline icd-1 & rps-0 & 497,806 & 493,798 & C56C10.8.1 & B0393.1.1 & 0.875 & 0.88 \\
\hline rla-1 & tpi-1 & 509,817 & 509,457 & Y37E3.7.2 & Y17G7B.7.2 & 0.501 & 0.501 \\
\hline icd-1 & rps-2 & 497,806 & 497,379 & C56C10.8.1 & С49H3.11.1 & 0.913 & 0.913 \\
\hline sod-2 & atp-2 & 498,735 & 496,479 & F10D11.1.1 & C34E10.6.3 & 0.271 & 0.595 \\
\hline hum-5 & $\operatorname{arx}-6$ & 506,256 & 496,567 & T02C12.1 & C35D10.16 & 0 & 0.421 \\
\hline rla-1 & eft-3 & 509,817 & 505,324 & Y37E3.7.2 & R03G5.1a.2 & 0.189 & 0.92 \\
\hline iff-1 & rpl-5 & 506,602 & 502,561 & T05G5.10 & F54C9.5.1 & 0.58 & 0.61 \\
\hline atp-2 & rpl-4 & 496,479 & 493,528 & C34E10.6.3 & B0041.4.1 & 0.828 & 0.835 \\
\hline rpl-5 & rps-13 & 502,561 & 495,308 & F54C9.5.1 & C16A3.9.1 & 0.999 & 0.999 \\
\hline unc-87 & unc-60 & 498,495 & 496,717 & F08B6.4a & C38C3.5b.1 & 0 & 0.407 \\
\hline mlc-3 & unc-87 & 498,666 & 498,495 & F09F7.2a.2 & F08B6.4a & 0.516 & 0.516 \\
\hline gpd-3 & atp-2 & 504,646 & 496,479 & K10B3.7.2 & C34E10.6.3 & 0.214 & 0.523 \\
\hline rpl-5 & rps-2 & 502,561 & 497,379 & F54C9.5.1 & С49H3.11.1 & 0.999 & 0.999 \\
\hline tct-1 & icd-1 & 499,979 & 497,806 & F25H2.11.2 & C56C10.8.1 & 0.746 & 0.751 \\
\hline rpl-1 & rps-2 & 511,889 & 497,379 & Y71F9AL.13a.1 & С49H3.11.1 & 0.798 & 0.995 \\
\hline pab-1 & icd-1 & 509,182 & 497,806 & Y106G6H.2a.4 & С56C10.8.1 & 0.458 & 0.458 \\
\hline rps-4 & rps-13 & 510,280 & 495,308 & Y43B11AR.4.2 & C16A3.9.1 & 0.999 & 0.999 \\
\hline rps-4 & rpl-7A & 510,280 & 509,604 & Y43B11AR.4.2 & Y24D9A.4a & 0.949 & 0.989 \\
\hline pab-1 & rpl-4 & 509,182 & 493,528 & Y106G6H.2a.4 & B0041.4.1 & 0.69 & 0.966 \\
\hline rps-17 & $\mathrm{rps}-0$ & 506,860 & 493,798 & T08B2.10.1 & B0393.1.1 & 0.999 & 0.999 \\
\hline rps-17 & icd-1 & 506,860 & 497,806 & T08B2.10.1 & C56C10.8.1 & 0.856 & 0.861 \\
\hline rpl-7A & drs-1 & 509,604 & 493,869 & Y24D9A.4a & B0464.1.1 & 0.451 & 0.519 \\
\hline rpl-18 & rla-1 & 510,406 & 509,817 & Y45F10D.12.2 & Y37E3.7.2 & 0.996 & 0.999 \\
\hline rpl-1 & rpl-18 & 511,889 & 510,406 & Y71F9AL.13a.1 & Y45F10D.12.2 & 0.832 & 0.993 \\
\hline daf-21 & unc-60 & 497,248 & 496,717 & C47E8.5.1 & C38C3.5b.1 & 0 & 0.911 \\
\hline sqv-8 & сур-31A2 & 512,932 & 503,484 & ZK1307.5 & H02I12.8 & 0.411 & 0.411 \\
\hline act-4 & rps-13 & 505,013 & 495,308 & M03F4.2a & C16A3.9.1 & 0.53 & 0.53 \\
\hline tct-1 & rps-13 & 499,979 & 495,308 & F25H2.11.2 & C16A3.9.1 & 0.996 & 0.996 \\
\hline crt-1 & eft-3 & 509,852 & 505,324 & Y38A10A.5.1 & R03G5.1a.2 & 0.573 & 0.573 \\
\hline rps-2 & daf-21 & 497,379 & 497,248 & С49H3.11.1 & C47E8.5.1 & 0.658 & 0.721 \\
\hline let-70 & сур-31A2 & 505,189 & 503,484 & M7.1 & H02I12.8 & 0 & 0.522 \\
\hline rps-13 & drs-1 & 495,308 & 493,869 & C16A3.9.1 & B0464.1.1 & 0.528 & 0.528 \\
\hline crt-1 & pdi-3 & 509,852 & 503,539 & Y38A10A.5.1 & H06О01.1.3 & 0.726 & 0.739 \\
\hline rpl-18 & rpl-7A & 510,406 & 509,604 & Y45F10D.12.2 & Y24D9A.4a & 0.925 & 0.984 \\
\hline rps-13 & rps-0 & 495,308 & 493,798 & C16A3.9.1 & B0393.1.1 & 0.999 & 0.999 \\
\hline rps-4 & rpl-4 & 510,280 & 493,528 & Y43B11AR.4.2 & B0041.4.1 & 0.999 & 0.999 \\
\hline
\end{tabular}


Table III. Continued.

\begin{tabular}{|c|c|c|c|c|c|c|c|}
\hline node 1 & node 2 & $\begin{array}{l}\text { node1_- } \\
\text { string_id }\end{array}$ & $\begin{array}{l}\text { node2_- } \\
\text { string_id }\end{array}$ & $\begin{array}{l}\text { node1__ } \\
\text { external_id }\end{array}$ & $\begin{array}{c}\text { node2_- } \\
\text { external_id }\end{array}$ & coexpression & $\begin{array}{l}\text { combined_ } \\
\text { score }\end{array}$ \\
\hline rps-4 & rps-2 & 510,280 & 497,379 & Y43B11AR.4.2 & С49H3.11.1 & 0.999 & 0.999 \\
\hline rla-1 & rpl-7A & 509,817 & 509,604 & Y37E3.7.2 & Y24D9A.4a & 0.872 & 0.912 \\
\hline rps-28 & rps-17 & 510,228 & 506,860 & Y41D4B .5.2 & T08B2.10.1 & 0.805 & 0.989 \\
\hline rpl-18 & tct-1 & 510,406 & 499,979 & Y45F10D.12.2 & F25H2.11.2 & 0.963 & 0.966 \\
\hline rla-2 & rps-28 & 511,588 & 510,228 & Y62E10A.1.1 & Y41D4B.5.2 & 0.799 & 0.879 \\
\hline eft-3 & rpl-4 & 505,324 & 493,528 & R03G5.1a.2 & B0041.4.1 & 0.713 & 0.97 \\
\hline tpi-1 & rpl-4 & 509,457 & 493,528 & Y17G7B.7.2 & В0041.4.1 & 0.489 & 0.498 \\
\hline tpi-1 & gpd-3 & 509,457 & 504,646 & Y17G7B.7.2 & K10B3.7.2 & 0.608 & 0.998 \\
\hline unc-54 & unc-60 & 498,853 & 496,717 & F11C3.3.1 & C38C3.5b.1 & 0 & 0.587 \\
\hline tpi-1 & cey-1 & 509,457 & 500,586 & Y17G7B.7.2 & F33A8.3.2 & 0 & 0.403 \\
\hline hum-5 & unc-54 & 506,256 & 498,853 & T02C12.1 & F11C3.3.1 & 0 & 0.54 \\
\hline rps-4 & pab-1 & 510,280 & 509,182 & Y43B11AR.4.2 & Y106G6H.2a.4 & 0.344 & 0.955 \\
\hline rla-2 & icd-1 & 511,588 & 497,806 & Y62E10A.1.1 & C56C10.8.1 & 0.809 & 0.81 \\
\hline rpl-7A & rpl-5 & 509,604 & 502,561 & Y24D9A.4a & F54C9.5.1 & 0.934 & 0.994 \\
\hline rps-17 & eft-3 & 506,860 & 505,324 & T08B2.10.1 & R03G5.1a.2 & 0.187 & 0.916 \\
\hline rpl-18 & rpl-4 & 510,406 & 493,528 & Y45F10D.12.2 & B0041.4.1 & 0.999 & 0.999 \\
\hline rla-2 & daf-21 & 511,588 & 497,248 & Y62E10A.1.1 & C47E8.5.1 & 0.38 & 0.548 \\
\hline rps-4 & tct-1 & 510,280 & 499,979 & Y43B11AR.4.2 & F25H2.11.2 & 0.941 & 0.946 \\
\hline rps-4 & rps-28 & 510,280 & 510,228 & Y43B11AR.4.2 & Y41D4B.5.2 & 0.76 & 0.971 \\
\hline rla-2 & rpl-5 & 511,588 & 502,561 & Y62E10A.1.1 & F54C9.5.1 & 0.967 & 0.999 \\
\hline rps-4 & rpl-5 & 510,280 & 502,561 & Y43B11AR.4.2 & F54C9.5.1 & 0.999 & 0.999 \\
\hline rla-1 & pab-1 & 509,817 & 509,182 & Y37E3.7.2 & Y106G6H.2a.4 & 0.29 & 0.929 \\
\hline rla-2 & rps-2 & 511,588 & 497,379 & Y62E10A.1.1 & С49H3.11.1 & 0.991 & 0.999 \\
\hline iff-1 & tct-1 & 506,602 & 499,979 & T05G5.10 & F25H2.11.2 & 0.483 & 0.483 \\
\hline rla-1 & rps-2 & 509,817 & 497,379 & Y37E3.7.2 & С49H3.11.1 & 0.997 & 0.999 \\
\hline rpl-1 & pab-1 & 511,889 & 509,182 & Y71F9AL.13a.1 & Y106G6H.2a.4 & 0.237 & 0.918 \\
\hline rps-28 & rps-2 & 510,228 & 497,379 & Y41D4B.5.2 & С49H3.11.1 & 0.732 & 0.981 \\
\hline rpl-5 & rpl-4 & 502,561 & 493,528 & F54C9.5.1 & B0041.4.1 & 0.999 & 0.999 \\
\hline rps-4 & rps-17 & 510,280 & 506,860 & Y43B11AR.4.2 & T08B2.10.1 & 0.995 & 0.999 \\
\hline rpl-18 & rps-17 & 510,406 & 506,860 & Y45F10D.12.2 & T08B2.10.1 & 0.956 & 0.996 \\
\hline rpl-1 & rpl-7A & 511,889 & 509,604 & Y71F9AL.13a.1 & Y24D9A.4a & 0.796 & 0.96 \\
\hline inf-1 & rps-0 & 503,072 & 493,798 & F57B9.6a.3 & B0393.1.1 & 0.414 & 0.433 \\
\hline alp-1 & unc-87 & 507,123 & 498,495 & T11B7.4d & F08B6.4a & 0.413 & 0.421 \\
\hline rla-1 & rpl-4 & 509,817 & 493,528 & Y37E3.7.2 & B0041.4.1 & 0.997 & 0.999 \\
\hline rps-2 & rps-13 & 497,379 & 495,308 & С49H3.11.1 & C16A3.9.1 & 0.998 & 0.999 \\
\hline pdi-3 & rps-2 & 503,539 & 497,379 & H06О01.1.3 & С49H3.11.1 & 0.457 & 0.457 \\
\hline cpl-1 & cpf-1 & 506,335 & 500,247 & T03E6.7.2 & F28C6.3 & 0 & 0.408 \\
\hline gyg-1 & cey-1 & 502,889 & 500,586 & F56B6.4a & F33A8.3.2 & 0 & 0.55 \\
\hline hum-5 & nmy-1 & 506,256 & 502,225 & T02C12.1 & F52B10.1 & 0 & 0.544 \\
\hline gpd-3 & sod-2 & 504,646 & 498,735 & K10B3.7.2 & F10D11.1.1 & 0 & 0.588 \\
\hline rps-4 & tpi-1 & 510,280 & 509,457 & Y43B11AR.4.2 & Y17G7B.7.2 & 0.444 & 0.444 \\
\hline rla-2 & trap-4 & 511,588 & 511,330 & Y62E10A.1.1 & Y56A3A.21.2 & 0.076 & 0.916 \\
\hline rps-28 & rpl-4 & 510,228 & 493,528 & Y41D4B.5.2 & B0041.4.1 & 0.756 & 0.847 \\
\hline drs-1 & rps-0 & 493,869 & 493,798 & B0464.1.1 & B0393.1.1 & 0.464 & 0.494 \\
\hline sdhd-1 & cyc-1 & 500,588 & 497,719 & F33A8.5.2 & C54G4.8.1 & 0.382 & 0.54 \\
\hline rpl-1 & icd-1 & 511,889 & 497,806 & Y71F9AL.13a.1 & С56C10.8.1 & 0.777 & 0.787 \\
\hline rps-17 & rps-13 & 506,860 & 495,308 & T08B2.10.1 & C16A3.9.1 & 0.997 & 0.999 \\
\hline rla-1 & rps-0 & 509,817 & 493,798 & Y37E3.7.2 & B0393.1.1 & 0.999 & 0.999 \\
\hline daf-21 & rpl-4 & 497,248 & 493,528 & C47E8.5.1 & B0041.4.1 & 0.59 & 0.634 \\
\hline tpi-1 & cyc-1 & 509,457 & 497,719 & Y17G7B.7.2 & C54G4.8.1 & 0.442 & 0.444 \\
\hline trap-4 & rpl-4 & 511,330 & 493,528 & Y56A3A.21.2 & B0041.4.1 & 0.308 & 0.951 \\
\hline
\end{tabular}


Table III. Continued.

\begin{tabular}{|c|c|c|c|c|c|c|c|}
\hline node 1 & node 2 & $\begin{array}{l}\text { node1_ } \\
\text { string_id }\end{array}$ & $\begin{array}{l}\text { node2_- } \\
\text { string_id }\end{array}$ & $\begin{array}{c}\text { node1__ } \\
\text { external_id }\end{array}$ & $\begin{array}{c}\text { node2_- } \\
\text { external_id }\end{array}$ & coexpression & $\begin{array}{l}\text { combined_ } \\
\text { score }\end{array}$ \\
\hline rpl-1 & rps-28 & 511,889 & 510,228 & Y71F9AL.13a.1 & Y41D4B.5.2 & 0.793 & 0.939 \\
\hline rps-28 & tct-1 & 510,228 & 499,979 & Y41D4B.5.2 & F25H2.11.2 & 0.683 & 0.683 \\
\hline eft-3 & $\mathrm{rpl}-5$ & 505,324 & 502,561 & R03G5.1a.2 & F54C9.5.1 & 0.701 & 0.974 \\
\hline sod-2 & cyc-1 & 498,735 & 497,719 & F10D11.1.1 & C54G4.8.1 & 0.456 & 0.604 \\
\hline pdi-3 & phy-2 & 503,539 & 500,798 & H06O01.1.3 & F35G2.4.1 & 0 & 0.4 \\
\hline rpl-18 & daf-21 & 510,406 & 497,248 & Y45F10D.12.2 & C47E8.5.1 & 0.387 & 0.401 \\
\hline rpl-1 & tct-1 & 511,889 & 499,979 & Y71F9AL.13a.1 & F25H2.11.2 & 0.751 & 0.757 \\
\hline rpl-1 & iff-1 & 511,889 & 506,602 & Y71F9AL.13a.1 & T05G5.10 & 0.612 & 0.624 \\
\hline rpl-1 & tpi-1 & 511,889 & 509,457 & Y71F9AL.13a.1 & Y17G7B.7.2 & 0.396 & 0.614 \\
\hline rps-0 & rpl-4 & 493,798 & 493,528 & B0393.1.1 & B0041.4.1 & 0.999 & 0.999 \\
\hline eft-3 & rps-13 & 505,324 & 495,308 & R03G5.1a.2 & C16A3.9.1 & 0.362 & 0.956 \\
\hline act-4 & unc-60 & 505,013 & 496,717 & M03F4.2a & C38C3.5b.1 & 0 & 0.863 \\
\hline crt-1 & pab-1 & 509,852 & 509,182 & Y38A10A.5.1 & Y106G6H.2a.4 & 0.425 & 0.453 \\
\hline tct-1 & atp-2 & 499,979 & 496,479 & $\mathrm{~F} 25 \mathrm{H} 2.11 .2$ & C34E10.6.3 & 0.575 & 0.583 \\
\hline rps-13 & rpl-4 & 495,308 & 493,528 & C16A3.9.1 & B0041.4.1 & 0.998 & 0.999 \\
\hline rps-2 & rpl-4 & 497,379 & 493,528 & С49H3.11.1 & B0041.4.1 & 0.999 & 0.999 \\
\hline rla-2 & rps-17 & 511,588 & 506,860 & Y62E10A.1.1 & T08B2.10.1 & 0.997 & 0.999 \\
\hline rla-1 & rps-17 & 509,817 & 506,860 & Y37E3.7.2 & T08B2.10.1 & 0.966 & 0.997 \\
\hline rps-17 & rps-2 & 506,860 & 497,379 & T08B2.10.1 & С49H3.11.1 & 0.969 & 0.999 \\
\hline cyc-1 & eat-6 & 497,719 & 493,780 & C54G4.8.1 & B0365.3.2 & 0.295 & 0.425 \\
\hline trap-4 & rpl-18 & 511,330 & 510,406 & Y56A3A.21.2 & Y45F10D.12.2 & 0.37 & 0.956 \\
\hline tpi-1 & rps-13 & 509,457 & 495,308 & Y17G7B.7.2 & C16A3.9.1 & 0.51 & 0.556 \\
\hline rla-2 & rps-0 & 511,588 & 493,798 & Y62E10A.1.1 & B0393.1.1 & 0.994 & 0.999 \\
\hline icd-1 & drs- 1 & 497,806 & 493,869 & C56C10.8.1 & B0464.1.1 & 0.403 & 0.509 \\
\hline sod-2 & $\mathrm{ftn}-2$ & 498,735 & 497,902 & F10D11.1.1 & D1037.3.4 & 0 & 0.494 \\
\hline pab-1 & cey-1 & 509,182 & 500,586 & Y106G6H.2a.4 & F33A8.3.2 & 0.317 & 0.447 \\
\hline pab-1 & rps-0 & 509,182 & 493,798 & Y106G6H.2a.4 & B0393.1.1 & 0.546 & 0.958 \\
\hline rla-1 & rpl-5 & 509,817 & 502,561 & Y37E3.7.2 & F54C9.5.1 & 0.996 & 0.999 \\
\hline rps-17 & rpl-4 & 506,860 & 493,528 & T08B2.10.1 & B0041.4.1 & 0.995 & 0.999 \\
\hline 1sm-3 & rpl-4 & 511,591 & 493,528 & Y62E10A.12.2 & B0041.4.1 & 0.126 & 0.622 \\
\hline tct-1 & rpl-4 & 499,979 & 493,528 & F25H2.11.2 & B0041.4.1 & 0.919 & 0.926 \\
\hline icd-1 & daf-21 & 497,806 & 497,248 & C56C10.8.1 & C47E8.5.1 & 0.439 & 0.478 \\
\hline rpl-1 & rps-0 & 511,889 & 493,798 & Y71F9AL.13a.1 & B0393.1.1 & 0.808 & 0.992 \\
\hline rpl-1 & trap-4 & 511,889 & 511,330 & Y71F9AL.13a.1 & Y56A3A.21.2 & 0.258 & 0.92 \\
\hline rps-4 & eft-3 & 510,280 & 505,324 & Y43B11AR.4.2 & R03G5.1a.2 & 0.402 & 0.939 \\
\hline rps-4 & rla-1 & 510,280 & 509,817 & Y43B11AR.4.2 & Y37E3.7.2 & 0.997 & 0.999 \\
\hline atp-2 & $\mathrm{rps}-0$ & 496,479 & 493,798 & C34E10.6.3 & B0393.1.1 & 0.432 & 0.763 \\
\hline rpl-7A & rps-2 & 509,604 & 497,379 & Y24D9A.4a & С49H3.11.1 & 0.914 & 0.978 \\
\hline rla-2 & rpl-7A & 511,588 & 509,604 & Y62E10A.1.1 & Y24D9A.4a & 0.936 & 0.958 \\
\hline rps-28 & icd-1 & 510,228 & 497,806 & Y41D4B.5.2 & C56C10.8.1 & 0.772 & 0.772 \\
\hline rpl-18 & icd-1 & 510,406 & 497,806 & Y45F10D.12.2 & С56C10.8.1 & 0.857 & 0.863 \\
\hline inf-1 & rps-2 & 503,072 & 497,379 & F57B9.6a.3 & С49H3.11.1 & 0.395 & 0.417 \\
\hline tpi-1 & tct-1 & 509,457 & 499,979 & Y17G7B.7.2 & F25H2.11.2 & 0.428 & 0.476 \\
\hline rps-17 & rpl-5 & 506,860 & 502,561 & T08B2.10.1 & F54C9.5.1 & 0.972 & 0.998 \\
\hline snr-2 & $\mathrm{cpf}-1$ & 508,903 & 500,247 & W08E3.1 & F28C6.3 & 0.245 & 0.919 \\
\hline pab-1 & rps-2 & 509,182 & 497,379 & Y106G6H.2a.4 & С49H3.11.1 & 0.704 & 0.968 \\
\hline skr-1 & pas-2 & 501,798 & 497,931 & F46A9.5.3 & D1054.2.1 & 0 & 0.899 \\
\hline rla-2 & act-4 & 511,588 & 505,013 & Y62E10A.1.1 & M03F4.2a & 0.508 & 0.508 \\
\hline icd-1 & rpl-4 & 497,806 & 493,528 & C56C10.8.1 & B0041.4.1 & 0.826 & 0.858 \\
\hline eft-3 & rps-2 & 505,324 & 497,379 & R03G5.1a.2 & С49H3.11.1 & 0.66 & 0.971 \\
\hline rpl-1 & rps-13 & 511,889 & 495,308 & Y71F9AL.13a.1 & C16A3.9.1 & 0.8 & 0.997 \\
\hline
\end{tabular}


Table III. Continued.

\begin{tabular}{|c|c|c|c|c|c|c|c|}
\hline node 1 & node 2 & $\begin{array}{l}\text { node1_- } \\
\text { string_id }\end{array}$ & $\begin{array}{l}\text { node2_- } \\
\text { string_id }\end{array}$ & $\begin{array}{c}\text { node1__ } \\
\text { external_id }\end{array}$ & $\begin{array}{c}\text { node2_- } \\
\text { external_id }\end{array}$ & coexpression & $\begin{array}{l}\text { combined } \\
\text { score }\end{array}$ \\
\hline rps-2 & atp-2 & 497,379 & 496,479 & С49H3.11.1 & C34E10.6.3 & 0.943 & 0.947 \\
\hline unc-54 & unc-87 & 498,853 & 498,495 & F11C3.3.1 & F08B6.4a & 0.566 & 0.776 \\
\hline tct-1 & rps-2 & 499,979 & 497,379 & $\mathrm{~F} 25 \mathrm{H} 2.11 .2$ & С49H3.11.1 & 0.999 & 0.999 \\
\hline trap-4 & rps-4 & 511,330 & 510,280 & Y56A3A.21.2 & Y43B11AR.4.2 & 0.245 & 0.921 \\
\hline rps-28 & iff-1 & 510,228 & 506,602 & Y41D4B.5.2 & T05G5.10 & 0.432 & 0.635 \\
\hline rla-1 & daf-21 & 509,817 & 497,248 & Y37E3.7.2 & C47E8.5.1 & 0.394 & 0.543 \\
\hline rps-4 & crt-1 & 510,280 & 509,852 & Y43B11AR.4.2 & Y38A10A.5.1 & 0.491 & 0.5 \\
\hline rpl-18 & crt-1 & 510,406 & 509,852 & Y45F10D.12.2 & Y38A10A.5.1 & 0.595 & 0.597 \\
\hline rps-2 & rps-0 & 497,379 & 493,798 & С49H3.11.1 & B0393.1.1 & 0.999 & 0.999 \\
\hline lev-11 & unc-87 & 509,147 & 498,495 & Y105E8B.1d & F08B6.4a & 0.272 & 0.454 \\
\hline trap-4 & rps-17 & 511,330 & 506,860 & Y56A3A.21.2 & T08B2.10.1 & 0.308 & 0.929 \\
\hline tct-1 & daf-21 & 499,979 & 497,248 & $\mathrm{~F} 25 \mathrm{H} 2.11 .2$ & C47E8.5.1 & 0.648 & 0.713 \\
\hline crt-1 & rpl-4 & 509,852 & 493,528 & Y38A10A.5.1 & B0041.4.1 & 0.472 & 0.604 \\
\hline rpl-18 & rps-4 & 510,406 & 510,280 & Y45F10D.12.2 & Y43B11AR.4.2 & 0.999 & 0.999 \\
\hline eft-3 & rps-0 & 505,324 & 493,798 & R03G5.1a.2 & B0393.1.1 & 0.523 & 0.959 \\
\hline rpl-1 & eft-3 & 511,889 & 505,324 & Y71F9AL.13a.1 & R03G5.1a.2 & 0.348 & 0.948 \\
\hline iff-1 & icd-1 & 506,602 & 497,806 & T05G5.10 & С56C10.8.1 & 0.47 & 0.47 \\
\hline rpl-7A & rps-13 & 509,604 & 495,308 & Y24D9A.4a & C16A3.9.1 & 0.944 & 0.979 \\
\hline eft-3 & ftn-2 & 505,324 & 497,902 & R03G5.1a.2 & D1037.3.4 & 0 & 0.999 \\
\hline rps-4 & drs-1 & 510,280 & 493,869 & Y43B11AR.4.2 & B0464.1.1 & 0.422 & 0.434 \\
\hline snr-2 & cey-1 & 508,903 & 500,586 & W08E3.1 & F33A8.3.2 & 0 & 0.899 \\
\hline rpl-1 & atp-2 & 511,889 & 496,479 & Y71F9AL.13a.1 & C34E10.6.3 & 0.144 & 0.403 \\
\hline rla-2 & rpl-4 & 511,588 & 493,528 & Y62E10A.1.1 & B0041.4.1 & 0.995 & 0.999 \\
\hline act-2 & act-4 & 506,426 & 505,013 & T04C12.5 & M03F4.2a & 0 & 0.481 \\
\hline cyn-7 & daf-21 & 512,151 & 497,248 & Y75B12B.2.2 & C47E8.5.1 & 0.359 & 0.408 \\
\hline rla-2 & tct-1 & 511,588 & 499,979 & Y62E10A.1.1 & F25H2.11.2 & 0.96 & 0.963 \\
\hline rps-4 & rps-0 & 510,280 & 493,798 & Y43B11AR.4.2 & B0393.1.1 & 0.999 & 0.999 \\
\hline rpl-18 & drs-1 & 510,406 & 493,869 & Y45F10D.12.2 & В0464.1.1 & 0.408 & 0.474 \\
\hline atp-2 & eat-6 & 496,479 & 493,780 & C34E10.6.3 & B0365.3.2 & 0.666 & 0.707 \\
\hline rpl-7A & rps-0 & 509,604 & 493,798 & Y24D9A.4a & В0393.1.1 & 0.942 & 0.947 \\
\hline lsm-3 & snr-2 & 511,591 & 508,903 & Y62E10A.12.2 & W08E3.1 & 0.323 & 0.625 \\
\hline rla-2 & rps-13 & 511,588 & 495,308 & Y62E10A.1.1 & C16A3.9.1 & 0.996 & 0.999 \\
\hline
\end{tabular}

rps- 4 and adenosine triphosphate synthase subunit $\beta$ mitochondrial precursor-2. Of note, the genes rpl-4, rps-13, rpl-5, rpl-1, rps-0, rla-1 and rpl-7A served critical roles in overall gene expression networks.

\section{Discussion}

The present study reported on the construction of a high-quality cDNA library from tissues of the earthworm Eisenia fetida (Savigny, 1826), following preliminary analysis of ESTs, putative functional analysis of the ESTs and the gene expression pattern associated with the physiological functions of this organism. cDNA libraries are widely used to identify genes and splice variants and are considered to be a physical resource for the construction of full-length clones $(18,19)$. In the present study, a cDNA library was utilized to provide a molecular resource for the analysis of genes involved in the specific biology of earthworms in terms of their development, survival, pathogenicity and virulence. There are two main factors to consider when assessing the quality of a cDNA library: Representation and cDNA lengths. According to Clarke-Carbon's formula (20), a cDNA library should contain at least $1.7 \times 10^{5}$ independent clones to ensure that $99 \%$ of low-abundance mRNAs will be represented in the library (21). Furthermore, the average length of the inserted cDNAs should be no less than $1.0 \mathrm{~kb}$ to ensure the integrity of cDNAs, indicating that in the present study the fragment sizes were effective for ensuring full-length cDNAs in the cDNA library. Since selection bias could favor the smaller cDNAs, the present study used fewer PCR cycles to minimize such bias as previously suggested (10). In addition, up to 25 PCR amplification cycles were used to generate an adequate amount of cDNA for cloning. 


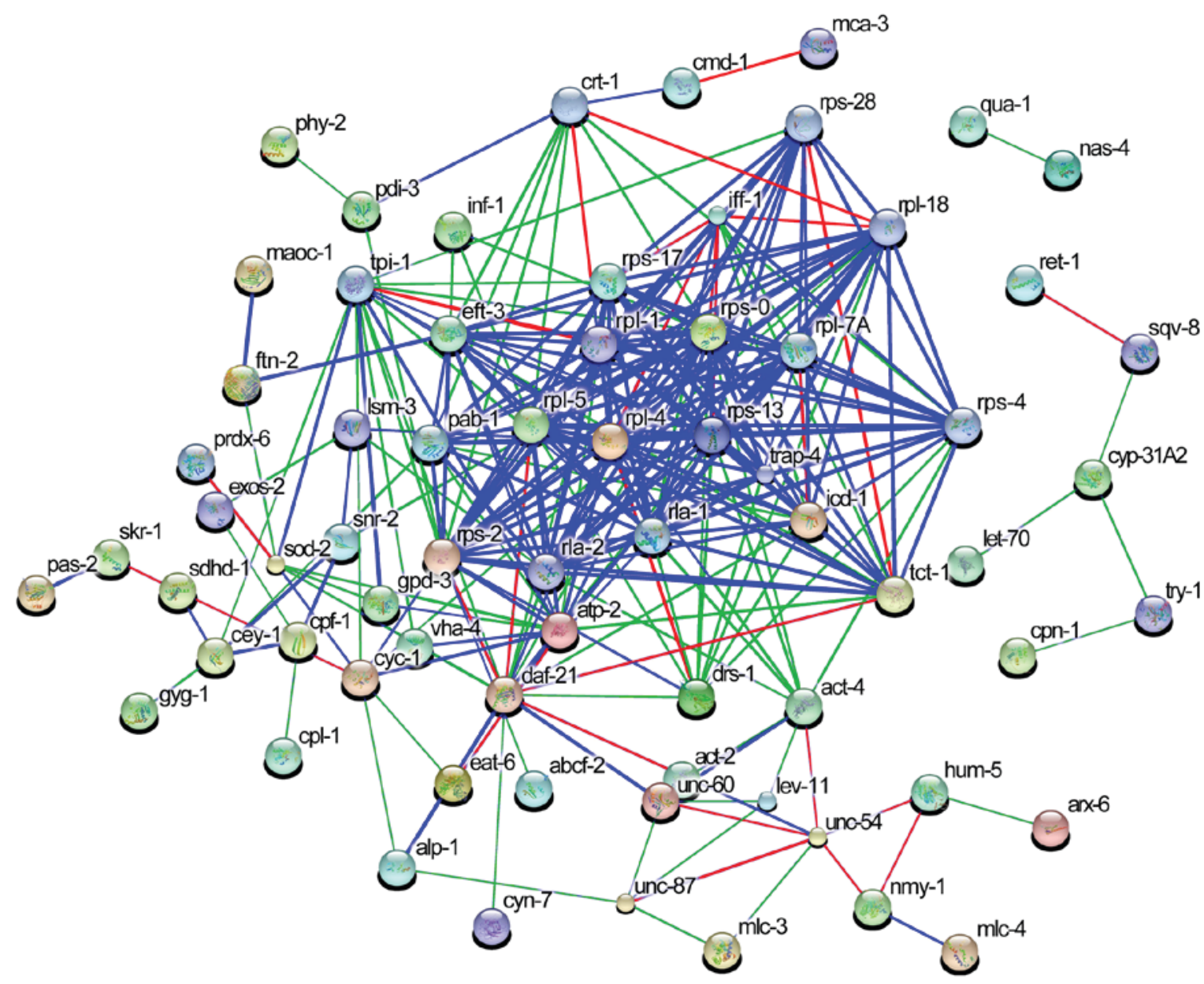

Figure 7. Connectivity between the predicted regulators and the clusters in the STRING network: Experimentally-derived interactions (green), co-expression (navy blue), and co-occurrences (red) in the genomes are shown. Colored circles represent input genes.

The generation of ESTs is an effective and unique approach in molecular studies as it allows for the analysis and measurement of gene expression, as well as simultaneous discovery of new genes. As each EST represents a copy of the functional part of a genome, the study of ESTs is believed to be a more effective way to discover functional genes (22). Furthermore, analysis of the expression of a large number of genes combined with the knowledge of their functions enables insight into the overall situation in terms of biological processes (23), for the current purposes in earthworms. In the present study, 91\% of the ESTs generated were sequences with known or putative functions, while the remainder represented unknown proteins or sequences with no similarities to those in the databases. Although close to 600 ESTs were reported, this is actually far from what could be considered as a 'complete' transcriptome (which usually includes between 15,000-20,000 to $>100,000$ ESTs). Therefore, the present characterization of this seemingly partial transcriptome may far from reflect the full transcriptomic profile of tissues in earthworms.

A comparison of the classification of ESTs with a C. elegans cDNA library based on their putative functions was conducted. Based on identification of clusters via GO analysis, 168 ESTs were matched to $C$. elegans genes by BLASTx. It is well known that earthworms serve significant roles in organic matter decomposition and mineral cycling, and thus are considered to be important contributors to soil fertility and humification processes (24). In the present study, hydrolytic enzyme activity, conjugating protein ligase activity, oxidation reduction and energy release activity of metabolic enzymes accounted for a large proportion of the molecular functions component. These molecular functions are considered to be a key part of the physiological functions of earthworms, which allow earthworms to survive in different soil environments.

Cell components, as part of GO annotation, are mainly categorized based on subcellular location (including cell cytoplasm, mitochondria, lysosomes, nucleus, microtubules, plasma membrane and myosin), which is highly important for the study of protein functions (25). The results of subcellular localization analysis can provide significant clues to aid the understanding of protein functions. In the present study, several cell components were determined through GO analysis of the 168 ESTs (annotated genes). It was evident that these cell components had strong associations with the regulation of gene expression during the biological development of earthworms, enabling the regeneration of the anterior portion, alterations in movement ability and tissue differentiation.

Due to the temporal specificity of gene expression and interactions with other gene products, the specific pathway undertaken, sequence of gene expression and expression pattern may ultimately change the effects of multiple pathways in 
earthworms (2). Therefore, the cDNA library and transcription profile of genes representative of fully developed adult earthworms may differ markedly to those of juvenile earthworms. In the present study, gene expression profiles representative of adult earthworm development were generated; however, gene expression profiling of juvenile earthworms was not performed, nor were analyses of the expected differential gene expression between juveniles and adults. In the present study, 168 individual ESTs of earthworms were analyzed in terms of KEGG pathway annotation, which identified 9 corresponding categories. Among them, glutathione metabolism is involved in antioxidant defense systems in Eisenia Andrei, and the associated enzymes are mainly identified in cytosolic fractions (26). Chondroitin sulfate and heparan sulfate biosynthesis are involved in biosynthesis pathways, which have important effects on growth and regeneration. In addition, heparan sulfate is also considered to be a type of anticoagulant, which may also be a function of arachidonic acid (27). Therefore, understanding the molecular function of earthworms may provide some basis for the treatment of thrombotic disorders.

Information on functional annotation and relevant biological interactions associated with a particular gene is available from many online resources. The gene network comprises a collection of genes that cooperate with each other to control the main biological processes. The STRING database suggested a functional context for earthworm lumbrokinase with unknown specificity. A previous study revealed that lumbrokinase and dilong administration can efficiently reduce the incidence of cardiac disease among nonsmokers exposed to second-hand smoke (28). In this regard, the discovery of genes and protein interactions in earthworms has provided a basis for further investigation into human diseases.

\section{Acknowledgements}

Not applicable.

\section{Funding}

The present study was supported by the National Natural Science Foundation of China (grant nos. 30472251, 30901821 and 81370312), the Natural Science Foundation for Young Scientists of Shanxi Province, China (grant no. 2010021035-2), Natural Science Foundation of Shanxi Province, China (grant no. 2015011113), International Scientific and Technological Cooperative Foundation of Shanxi Province, China (grant no. 201703D421023), the Fund for Shanxi '1331 Project' Key Subjects Construction and the Fund for Shanxi Key Subjects Construction.

\section{Availability of data and materials}

The datasets used and/or analyzed during the current study are available from the corresponding author on reasonable request.

\section{Authors' contributions}

BFY, BN and JX conceived and designed the experiments and bioinformatics analysis. CL and FXM performed bioinformatics analysis, and wrote the manuscript. XW, PYM, QZ and
JBT performed the experiments. RG, ZZL, HLW and NLC contributed to designing the present study and revising the manuscript. JHW and GQS analyzed and interpreted the data.

\section{Ethics approval and consent to participate}

Not applicable.

\section{Patient consent for publication}

Not applicable.

\section{Competing interests}

The authors declare that they have no competing interests.

\section{References}

1. Torres-Leguizamon M, Mathieu J, Decaens T and Dupont L: Genetic structure of earthworm populations at a regional scale: Inferences from mitochondrial and microsatellite molecular markers in Aporrectodea icterica (Savigny 1826). PLoS One 9: e101597, 2014.

2. Pirooznia M, Gong $\mathrm{P}$, Guan X, Inouye LS, Yang K, Perkins EJ and Deng Y: Cloning, analysis and functional annotation of expressed sequence tags from the Earthworm Eisenia fetida. BMC Bioinformatics 8 (Suppl 7): S7, 2007.

3. Cho SJ, Koh KS, Lee E and Park SC: Differential expression of threelabial genes during earthworm head regeneration. Biosci Biotechnol Biochem 73: 2609-2614, 2014.

4. Zheng P, Shao Q, Diao X, Li Z and Han Q: Expression of stem cell pluripotency factors during regeneration in the earthworm Eisenia foetida. Gene 575: 58-65, 2016.

5. Kim TH, Kim NS, Lim D, Lee KT, Oh JH, Park HS, Jang GW, Kim HY, Jeon M, Choi BH, et al: Generation and analysis of large-scale expressed sequence tags (ESTs) from a full-length enriched cDNA library of porcine backfat tissue. BMC Genomics 7: 36, 2006.

6. Wellenreuther R, Schupp I, Poustka A and Wiemann S; German cDNA Consortium: SMART amplication combined with cDNA size fractionation in order to obtain large full-length clones. BMC Genomics 5: 36, 2004.

7. Dai Y, Su W, Yang C, Song B, Li Y and Fu Y: Development of novel polymorphic EST-SSR markers in bailinggu (Pleurotus tuoliensis) for crossbreeding. Genes (Basel) 8: pii: E325, 2017.

8. Liu C, Liu D, Guo Y, Lu T, Li X, Zhang M, Ma J, Ma Y and Guan W: Construction of a full-length enriched cDNA library and preliminary analysis of expressed sequence tags from Bengal Tiger Panthera tigris tigris. Int J Mol Sci 14: 11072-11083, 2013.

9. Carninci P, Shibata Y, Hayatsu N, Itoh M, Shiraki T, Hirozane T, Watahiki A, Shibata K, Konno H, Muramatsu M and Hayashizaki Y: Balanced-size and long-size cloning of full-length, cap-trapped cDNAs into vectors of the novel lambda-FLC family allows enhanced gene discovery rate and functional analysis. Genomics 77: 79-90, 2001.

10. Ling $\mathrm{P}$, Wang $\mathrm{M}$, Chen $\mathrm{X}$ and Campbell KG: Construction and characterization of a full-length cDNA library for the wheat stripe rust pathogen (Puccinia striiformis f. sp. tritici). BMC Genomics 8: 145, 2007.

11. Ye J, McGinnis S and Madden TL: BLAST: Improvements for better sequence analysis. Nucleic Acids Res 34 (Web Server Issue): W6-W9, 2006.

12. NCBI Resource Coordinators: Database resources of the national center for biotechnology information. Nucleic Acids Res 41: (Database Issue): D8-D20, 2013.

13. Thompson JD, Higgins DG and Gibson TJ: CLUSTAL W: Improving the sensitivity of progressive multiple sequence alignment through sequence weighting, position-specific gap penalties and weight matrix choice. Nucleic Acids Res 22: 4673-4680, 1994.

14. Wang J, Duncan D, Shi Z and Zhang B: WEB-based GEne SeT AnaLysis Toolkit (WebGestalt): Update 2013. Nucleic Acids Res 41 (Web Server Issue): W77-W83, 2013. 
15. Zhang B, Kirov S and Snoddy J: WebGestalt: An integrated system for exploring gene sets in various biological contexts. Nucleic Acids Res 33 (Web Server Issue): W741-W748, 2005.

16. Ravaux J, Hassanin A, Deutsch J, Gaill F and MarkmannMulisch U: Sequence analysis of the myosin regulatory light chain gene of the vestimentiferan Riftia pachyptila. Gene 263: 141-149, 2001.

17. Kanehisa M, Goto S, Kawashima S and Nakaya A: The KEGG databases at GenomeNet. Nucleic Acids Res 30: 42-46, 2002.

18. Wiemann S, Mehrle A, Bechtel S, Wellenreuther R, Pepperkok R and Poustka A; German cDNA Consortium: cDNAs for functional genomics and proteomics: The German Consortium. Comptes Rendus Biologies 326: 1003-1009, 2003.

19. Blair MW, Fernandez AC, Ishitani M, Moreta D, Seki M, Ayling S and Shinozaki K: Construction and EST sequencing of full-length, drought stress cDNA libraries for common beans (Phaseolus vulgaris L.). BMC Plant Biol 11: 171, 2011.

20. Clarke L and Carbon J: A colony bank containing synthetic CoI EI hybrid plasmids representative of the entire E. coli genome. Cell 9: 91-99, 1976.

21. Li YP, Xia RX, Wang H, Li XS, Liu YQ, Wei ZJ, Lu C and Xiang ZH: Construction of a full-length cDNA Library from Chinese oak silkworm pupa and identification of a KK-42-binding protein gene in relation to pupa-diapause termination. Int J Biol Sci 5: 451-457, 2009.

22. Hatey F, Tosser-Klopp G, Clouscard-Martinato K, Mulsant P and Gasser F: Expressed sequence tags for genes: A review. Genet Selec Evol 30: 521-524, 1998.
23. Thanh T, Chi VT, Abdullah MP, Omar H, Noroozi M, Ky H and Napis S: Construction of cDNA library and preliminary analysis of expressed sequence tags from green microalga Ankistrodesmus convolutus Corda. Mol Biol Rep 38: 177-182, 2011.

24. Duo L, Yin L, Zhang C and Zhao S: Ecotoxicological responses of the earthworm Eisenia fetida to EDTA addition under turfgrass growing conditions. Chemosphere 220: 56-60, 2018.

25. Ashburner M, Ball CA, Blake JA, Botstein D, Butler H, Cherry JM, Davis AP, Dolinski K, Dwight SS, Eppig JT, et al: Gene ontology: Tool for the unification of biology. The Gene Ontology Consortium. Nat Genet 25: 25-29, 2000.

26. Homa J, Stalmach M, Wilczek G and Kolaczkowska E: Effective activation of antioxidant system by immune-relevant factors reversely correlates with apoptosis of Eisenia andrei coelomocytes. J Comp Physiol B 186: 417-430, 2016.

27. Li P, Sheng J, Liu Y, Li J, Liu J and Wang F: Heparosan-derived heparan sulfate/heparin-like compounds: One kind of potential therapeutic agents. Med Res Rev 33: 665-692, 2013.

28. Lai CH, Han CK, Shibu MA, Pai PY, Ho TJ, Day CH, Tsai FJ, Tsai CH, Yao CH and Huang CY: Lumbrokinase from earthworm extract ameliorates second-hand smoke-induced cardiac fibrosis. Environ Toxicol 30: 1216-1225, 2015.

This work is licensed under a Creative Commons Attribution-NonCommercial-NoDerivatives 4.0 International (CC BY-NC-ND 4.0) License. 